\title{
microRNA expression profiling as supportive diagnostic and therapy prediction tool in chronic myeloid leukemia
}

\author{
D. JURKOVICOVA ${ }^{1, *}$, R. LUKACKOVA ${ }^{2}$, M. MAGYERKOVA ${ }^{1}$, L. KULCSAR ${ }^{1}$, M. KRIVJANSKA ${ }^{1}$, V. KRIVJANSKY ${ }^{1}$, M. CHOVANEC ${ }^{1,3}$
}

${ }^{1}$ KRD Molecular Technologies Ltd., Saratovska 26, 84102 Bratislava, Slovakia; ${ }^{2}$ MEDIREX a. s., Galvaniho 17/C, 82016 Bratislava, Slovakia; ${ }^{3}$ Department of Genetics, Cancer Research Institute, Slovak Academy of Sciences, Vlarska 7, 83391 Bratislava, Slovakia

${ }^{*}$ Correspondence: dana.j@krdlab.sk

Received May 20, 2015 / Accepted July 27, 2015

Chronic myeloid leukemia (CML) is a myeloproliferative disorder of hematopoietic stem cells carrying Philadelphia $(\mathrm{Ph})$ chromosome and the oncogenic BCR-ABL1 fusion gene. microRNAs (miRNAs) belong to hematopoiesis transcription regulators and their deregulated expression associates with pathogenesis of CML. The current study assesses and validates expression profiles of selected oncogenic and tumor suppressing miRNAs that are associated with different imatinib mesylate (IM) response in CML patients carrying rare $B C R-A B L$ variants. Microarray analysis has identified different expression of 70 miRNAs (46 up- and 24 down-regulated) when compared IM-resistant with IM-responsive patients carrying Ph chromosome. Significantly up-regulated expression of oncogenic miRNAs (miR-17, miR-18a, miR-20a, miR-21, miR-27a and miR-155) and significantly down-regulated expression of tumor supressing mRNAs (let-7d, miR-205, miR-320, miR-451 and miR-574) in IM-resistant compared to IM-responsive patients was confirmed and validated by qRT-PCR. This study confirms the involvement of the selected oncogenic and tumor suppressing miRNAs in CML pathogenesis and IM response and suggests that these miRNAs could be suitable biomarkers for differential diagnosis of CML patients carrying rare $B C R-A B L$ transcripts, as well as for prediction of their IM response and therapy outcome.

Key words: miRNA, chronic myeloid leukemia, microarray, qRT-PCR, peripheral blood

\footnotetext{
Abbreviations: ABL - Abelson oncogene; AML - acute myeloid leukemia; BCR - breakpoint cluster region; BCR-ABL1 - fusion gene; BIM - pro-apoptotic member of the BCL-2 protein family; CD $34^{+}$cells - cells expressing hematopoietic progenitor cell antigen CD34; CEBPA - CCAAT/enhancer-binding protein alpha; c-Jun - c-Jun N-terminal kinase; CLL - chronic lymphocytic leukemia; $\mathrm{CML}$ - chronic myeloid leukemia; dATP - deoxyadenosine triphosphate; dCTP - deoxycytidine triphosphate; dGTP - deoxyguanosine triphosphate; DLBCL - diffuse large B-cell lymphoma; dTTP - deoxythymidine triphosphate; E2F1 - transcription factor E2F1; E2F3 - transcription factor E2F3; EMT - epithelial to mesenchymal transition; ERK - extracellular-signal-regulated kinase; IM imatinib mesylate; K562 cells - human immortalized myelogenous leukemia cell line; let 7 - microRNA let-7 family; MAPK - mitogen-activated protein kinases; miR-17 92 cluster - cluster consisting of miR-17, miR-18a, miR-19a, miR-20a, miR-19b-1 and miR-92a-1; miRNAs - microRNAs; MuLV reverse transcriptase - Murine Leukemia Virus reverse transcriptase; MYC - MYC proto-oncogene protein; NF- $\mathrm{KB}$ - nuclear factor kappa-light-chain-enhancer of activated B cells; p38 MAPK - pP38 mitogen-activated protein kinases; PDCD4 - programmed cell death protein 4; $\mathrm{Ph}$ chromosome/Ph+ - Philadelphia chromosome; PTEN - phosphatase and tensin homolog; PU.1 - transcription factor; qRT-PCR - quantitative reverse transcription and polymerase chain reaction; RNA - ribonucleic acid; RT - reverse transcription; RUNX1 - Runt-related transcription factor 1; Snord38B - small nucleolar RNA U38B; Snord44 - small nucleolar RNA U44; STAT5 - signal transducer and activator of transcription 5; TfR-1 - transferrin receptor-1; UTRs - untranslated regions.
}

Chronic myeloid leukemia (CML) is a myeloproliferative disorder characterized by expansion of a clone of hematopoietic cells that carry the Philadelphia $(\mathrm{Ph})$ chromosome [1]. The $\mathrm{Ph}$ chromosome is a single reciprocal chromosomal translocation $\mathrm{t}(9 ; 22)(\mathrm{q} 34 ; \mathrm{q} 11)$ between the long arms of chromosome 9 and 22, resulting in the fusion of the Abelson $(A B L)$ oncogene to the breakpoint cluster region $(B C R)$ gene. This translocation results in the formation of a hybrid $B C R-A B L$ oncogene on chromosome 22 , which codes for a deregulated and constitutively activated tyrosine kinase, which is involved in pathogenesis of CML [2]. BCR-ABL activates multiple signal transduction pathways, including mitogen-activated protein kinase, phosphatidylinositol 3 kinase, STAT5/Janus kinase, and MYC, leading to uncontrolled cell proliferation, reduced apoptosis and resulting malignant expansion of pluripotent stem cells in bone marrow [3].

Imatinib mesylate (IM) is a targeted tyrosine kinase inhibitor, the gold standard of CML therapy, inducing a complete hematological and cytogenetic response in $80 \%$ of newly diagnosed CML patients. However, primary resistance to IM and 
molecular evidence of persisting disease have been observed in $20-25 \%$ of IM-treated patients [4].

Molecular diagnostics of CML using qRT-PCR and nested PCR enables to confirm the $B R C-A B L$ fusion gene and determines the number of $B C R-A B L$ transcripts with very good correlation between blood and bone marrow samples. In $95 \%$ of CML cases, $B C R-A B L$ fusion transcripts are $\mathrm{b} 2 \mathrm{a} 2$ and $\mathrm{b} 3 \mathrm{a} 2$ and these can be detected by qRT-PCR. In the rest of CML patients, rare b3a3, b2a3, c3a2, b1a1 and e1a3 or other variants are typical, but these are undetectable by routine diagnostic qRT-PCR and can only be revealed by non-quantitative PCR. These rare $B C R-A B L$ variants occur in exceptional CML cases where $\mathrm{BCR}$ breakpoints are outside of the main defined cluster regions (major-M-BCR, minor-m-BCR or micro- mu-BCR), or with unusual breakpoints in $A B L$ resulting in $B C R-A B L$ transcripts with b2a3 or b3a3 junctions, or with aberrant fusion transcripts containing variable lengths of intronic sequence inserts. Different types of $B C R-A B L$ rearrangement are associated with different clinical course and prognosis and their frequency in population relates with different ethnic backgrounds $[5,6]$. Consequently, also the treatment outcomes are difficult to monitor. In such cases, microRNA (miRNA) expression profiling could provide reliable alternative diagnostic and predictive tool to confirm CML diagnosis and predict IM-resistance.

Chromosomal translocations are usually associated with amplification or deletion of regions carrying sequences encoding important regulatory molecules, including miRNAs. miRNAs are non-coding, small (20-25 nts) single-stranded RNA molecules that regulate the expression of genes involved in cell development, proliferation [7] and differentiation [8], apoptosis [9], immune response, inflammation [10], viral infection [11], and also in pathological states such as autoimmune, cancerous [12] and other diseases.

miRNAs have been shown to promote tumor growth acting both as oncogenes and tumor suppressors. Aberrant miRNA levels have been observed in many cancers, as compared to their normal tissue counterparts [13]. The first miRNA molecules associated with human leukemia pathogenesis have been found in chronic lymphocytic leukemia (CLL) [14]. Deletion of chromosome (13)(q14) in CLL has been

Table 1. IM-response and $B C R-A B L$ variants of patients.

\begin{tabular}{ccc}
\hline Patient & $\boldsymbol{B C R}$ - $\boldsymbol{A} \boldsymbol{B L}$ variant & IM - response \\
\hline 1 & $\mathrm{c} 3 \mathrm{a} 2$ & resistant \\
2 & $\mathrm{~b} 3 \mathrm{a} 3$ & resistant \\
3 & $\mathrm{~b} 3 \mathrm{a} 3$ & resistant \\
4 & $\mathrm{~b} 2 \mathrm{a} 3$ & resistant \\
5 & $\mathrm{~b} 2 \mathrm{a} 3$ & responsive \\
6 & $\mathrm{~b} 2 \mathrm{a} 3$ & responsive \\
7 & $\mathrm{e} 1 \mathrm{a} 3$ & responsive \\
8 & $\mathrm{~b} 1 \mathrm{a} 1$ & responsive \\
9 & $\mathrm{~b} 3 \mathrm{a} 3$ & responsive \\
\hline
\end{tabular}

linked to down-regulation of suppressors miR-15a and miR16 $[14,15]$. In contrast, in B-cell lymphomas amplification of chromosome (13)(q31) is associated with overexpression of the oncogenic miRNA cluster 17 92 [16]. Although several deregulated miRNAs have already been associated with CML, some data is rather controversial and its relevant diagnostic and predictive value is still missing. Oncogenic miRNAs, miR155, miR-17 92 cluster, miR-15a, miR-16, miR-142, miR-181, miR-221 and some others, have been described as crucial regulatory molecules associated with hematomalignancies, including CML. Their deregulation has been observed in acute myeloid leukemia (AML), CLL, diffuse large B-cell lymphoma (DLBCL) [17-19], and in a number of leukemia and lymphoid cell lines [13, 15, 19-25]. In present study, we have focused on validation of the selected miRNAs identified as the most relevant for CML diagnosis confirmation and early IM response prediction. To achieve this, we employed a microarray platform to characterize the group of differentially expressed miRNAs in peripheral blood mononuclear cells from IM-resistant and IM-responsive patients carrying $\mathrm{Ph}$ chromosome. Of the group of differentially expressed miRNAs, we have further focused on up-regulated oncogenic miRNAs, miR-17 92 cluster (namely miR-17, miR-18a, and miR-20a), miR-21, miR-27a and miR-155, as well as on down-regulated tumor suppressing miRNAs, let-7d, miR205, miR-320, miR-451 and miR-574. Expression profiles of these mRNAs were subsequently validated by qRT-PCR. We suggest that, in addition to well-known gene mutations and aberrations, deregulated expression profiles of these miRNAs can provide further molecular tool for CML diagnosis and therapy prediction. Hence, miRNA profiling can clinically be used for CML diagnosis, patient stratification, treatment response prediction and therapy outcome monitoring, especially in rare cases where conventional molecular diagnostics is not sufficient and/or is unable.

\section{Patients and methods}

Patient samples. Blood samples were obtained from 9 CML patients with detected $\mathrm{Ph}$ chromosome ( $\mathrm{Ph}+$ patients) and 14 healthy individuals in the Clinics of Hematology and Transfusion, University Hospital of Medical Faculty of Comenius University, and in National Institute for Oncology, Bratislava, Slovakia. The retrospective patient study identified $B R C-A B L$ positive cases with fusion atypical transcripts - c3a2, b1a1, b3a3, b2a3 and ela3. Selection of patients with rare $B R C-A B L$ fusion transcripts explains the small cohort analyzed in this study. CML diagnosis was determined by conventional diagnostic methods. Patients were treated with imatinib mesylate (IM; dose $400 \mathrm{mg} /$ day) during 6 months. In this study, according to therapy outcome, 5 patients identified as IM-responsive and 4 patients as IM-resistant were further analyzed for miRNA expression profiles (Table 1).

The study was approved by the scientific committee of the University Hospital of Medical Faculty of Comenius 
University and National Institute for Oncology, Bratislava Slovakia. All subjects donated their samples with informed consent approved by the Ethic Committee of the Institute of Hematology and Blood Transfusion and each patient/donor gave written informed consent in accordance with the Declaration of Helsinki. Peripheral blood mononuclear cells were prepared by sedimentation using Ficoll-Paque ${ }^{\mathrm{TM}}$ PLUS (GE Healthcare, Freiburg, Germany) density gradient. Cells were suspended in freezing solution (DMEM supplemented with $10 \%$ DMSO and $20 \%$ fetal calf serum) and stored at $-80{ }^{\circ} \mathrm{C}$ before RNA extraction.

RNA extraction. To extract total RNA containing all small RNA molecules including miRNAs, TRI Reagent solution (Life technologies), Ribo Pure-Blood kit (Ambion Inc., no. AM1928, Austin, TX, USA) and RNA/DNA/Protein Purification Kit (Norgen Biotek ${ }^{\circledR}$, Thorold, ON, Canada) were used. Total RNA was quantified using the NanoDrop ND-1000 Spectrophotometer (Thermo Scientific, USA) and the Qubit fluorimeter (Qubit ${ }^{\circ}$ RNA HS Assay Kit, LifeTechnologies, USA). The RNA integrity was assessed using the Agilent 2100 bioanalyzer (Agilent, Palo Alto, CA, USA). Ribo Pure-Blood kit and RNA purification kit gave better 230/280 ratio, and therefore were used for RNA extraction for microarrays. TRI Reagent RNA extraction was used for real-time qRT-PCR reactions.

miRNA microarray profiling and data analysis. miRNA expression profiling in patients' samples performed using $100 \mathrm{ng}$ of total RNA was processed using the Agilent miRNA Complete Labeling and Hyb Kit (5190-0456, Santa Clara, California, USA) according to the manufacturer's protocol (version 2.4, September 2011) and recommendations. Briefly, after the dephosphorylation of the $3^{\prime}$ ends of RNA molecules, the Cyanine3-pCp was ligated to the $3^{\prime}$ end. Next, the samples were dried, suspended in the hybridization mix and loaded onto the SUREPRINT G3 UNRESTRICTED MIRNA 8X60K slide (G4872A-04606). The loaded slide, placed in the Agilent Microarray Hybridization Chamber (G2534), was incubated for $20 \mathrm{hrs}$ at $55^{\circ} \mathrm{C} / 20 \mathrm{rpm}$ in the Agilent hybridization oven (G2545A). After washing with the Gene Expression Wash Buffers 1 and 2 (5188-5327), the washed and dried slide was scanned in the Innoscan 900 microarray scanner (Innopsys Inc., Carbonne, France) with the resolution of 3 micrometers. The resulting images of individual arrays were extracted with the Mapix 7.3.0 software (Innopsys Inc., Carbonne, France) and exported as GPR files.

GPR files were imported into the R workspace by the read. maimages() function in the limma package and transformed into the "uRNAList" format to enable use of functions from the R package "AgiMicroRna“. Subsequently, without background substraction, data were quantille-normalized between arrays by the function rmaMicroRna()in the AgiMicroRna package. Finally, standard limma procedure was applied to the normalized data to analyze differential expression between studied groups.

miRNA qRT-PCR quantification. Differentially expressed oncogenic miRNAs, miR-17, miR-18a, miR-20a, miR-21,
miR-27a, miR-155, and tumor suppressing miRNAs, let7d, miR-205, miR-320, miR-451 and miR-574, identified by miRNA microarray were further validated by qRT-PCR. Expression of mature miRNAs was determined using FirstStrand cDNA Synthesis System (Central European Biosystems, Czech Republic) supplemented with poly(A)polymerase (Takara, Japan) and ATP (Sigma, Germany). Briefly, for cDNA synthesis $100 \mathrm{ng}$ of RNA in a final volume of $10 \mu$ including $1 \mu \mathrm{l}$ of $10 \mathrm{x}$ poly(A)polymerase buffer, $0.1 \mathrm{mM}$ of ATP, $1 \mu \mathrm{M}$ of RT-primer, $0.1 \mathrm{mM}$ of each deoxynucleotide (dATP, dCTP, dGTP and dTTP), 100 units of MuLV reverse transcriptase and 1 unit of poly(A)polymerase was incubated at $42^{\circ} \mathrm{C}$ for 1 hour followed by enzyme inactivation at $95^{\circ} \mathrm{C}$ for 5 minutes. The sequence of the oligo- $\mathrm{d}(\mathrm{T}) / \mathrm{adapter}$ primer was 5'-CAGGTCCAGTTTTTTTTTTTTTTTVN, where $\mathrm{V}$ is A, C and $\mathrm{G}$ and $\mathrm{N}$ is $\mathrm{A}, \mathrm{C}, \mathrm{G}$ and $\mathrm{T}$ (IDT, Leuven, Belgium).

Real-time PCR detection and quantification of mature forms of hsa-miR-17-5p, hsa-miR-18a-5p, hsa-miR-20a-5p, hsa-miR-21-5p, hsa-miR-27a-3p, hsa-miR-155-5p, hsa-let7d-3p, hsa-miR-205-3p, hsa-miR-320-5p, hsa-miR-451-5p, hsa-miR-574-5p and small RNA reference endogenous controls Snord38B and Snord44 were performed using SYBR Premix Ex Taq II (Tli RNaseH Plus), ROX plus (Takara, Japan), adapter-specific reverse primer and miRNA-specific (and Snord-specific) forward primers (Utility model 6932 and Utility model applications PUV5039/2014-5045/2014). qPCR was performed using BIOER, LineGene 9660 Real-Time PCR System (Hangzhou Bioer Technology Co., Ltd, China) at following settings: $95^{\circ} \mathrm{C}$ for $5 \mathrm{~min}$, followed by 40 cycles of $95^{\circ} \mathrm{C}$ for $20 \mathrm{sec}$ and $60^{\circ} \mathrm{C}$ for $50 \mathrm{sec}$, followed by melt cycle. Mature miRNA amplification was done in triplicate. Ct values were averaged and normalized against reference endogenous controls Snord44 and/or Snord38B. Relative expression was determined by the $2^{-(\Delta \Delta \mathrm{Ct})}$ comparative threshold method.

Statistical analysis. The significance of fold change in miRNA expression was analyzed using the Wilcoxon signed rank test applied to the $\Delta \mathrm{Ct}$ values. miRNA differential expression analysis between unrelated samples was conducted using the 2-tailed Mann-Whitney U test. Analyses were performed using GraphPad Prism v.5 (La Jolla, CA, USA) and SAS v.9 (Cary, NJ, USA) Software. Results with a $P$ value $<0.05$ were considered significant.

\section{Results}

Microarray miRNA expression profiles and IM-response. To decipher a potential miRNA expression signature associated with IM response in CML using a miRNA microarray, we have analyzed expression profiles of miRNAs of $\mathrm{Ph}+$ $\mathrm{IM}$-resistant patients and compared them with $\mathrm{Ph}+$ patients who responded to drug. Microarray analysis identified 70 differently expressed miRNAs between IM-resistant and IM-responsive patients. The expression levels of miR-17, miR-18a, miR-19a, miR-20a (those of miR-17 92 cluster), miR-21, miR-27a and miR-155, have markedly been increased 

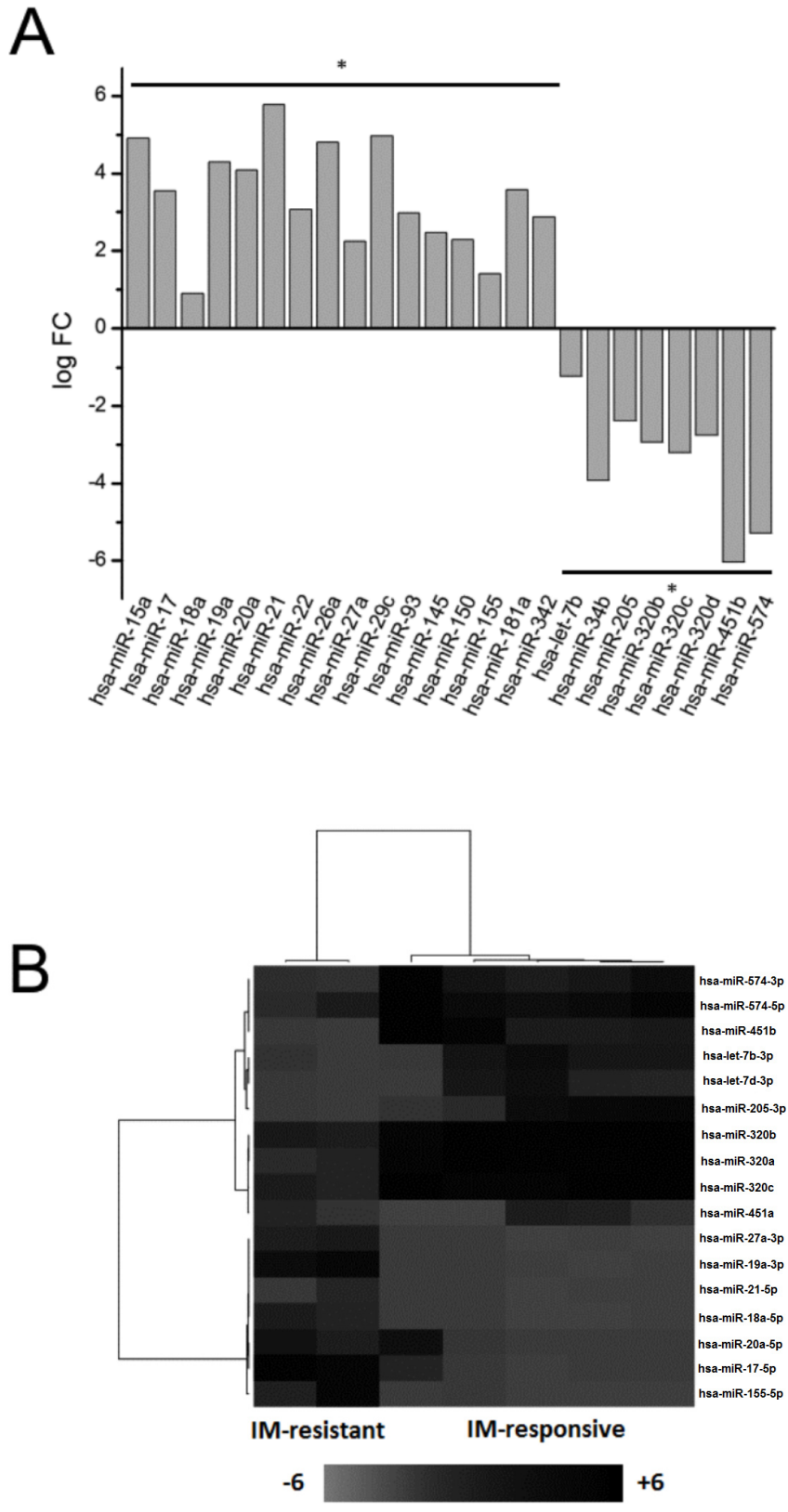

Figure 1. miRNA microarray expression profile and cluster analysis of IM-resistant against IM-responsive Ph+ patients. A) Log FC values showed up-regulation of oncogenes miR-17, miR-18a, miR-19a, and miR-20a of miR-17 92 cluster, next miR-21, miR-22, miR-27a, miR26a, miR-29c, miR-93, miR-145, mir-155, miR-181a and miR-342, and down-regulation of tumor suppressors let-7, miR-34b, miR-205, miR-320b-d, miR451b and miR-574 in IM-resistant compared to IMresponsive $\mathrm{Ph}+$ patients. Values represent mean $\pm S E M ; n=4,{ }^{\star} \mathrm{p}<0.05$. B) Cluster analysis confirmed differential expression of the selected miRNA oncogenes and tumor suppressors between IM-resistant and IM-responsive $\mathrm{Ph}+$ patients. Shown is representative miRNA array heat map where miRNA expression profiles of 2 IM-resistant patients were compared with those of 4 IM-responsive patients. Performed Agilent miRNA arrays use the quantile normalization with no background subtraction (transformation), as recommended by manufacturer (light grey scale - down-regulated expression, dark grey/black scale up-regulated expression). in IM-resistant compared to IM-responsive $\mathrm{Ph}+$ patients. In addition, expression levels of miR-15a, miR-22, miR-26a/b, miR-29c, miR-93, miR-145, miR-150, miR-181a and miR342 were moderately increased in IM-resistant $\mathrm{Ph}+$ patients. Let-7b, d, miR-34b, miR-205, miR-320b, c, d, miR-451b and miR-574 were the most down-regulated miRNAs. Based on this data, all above expression profiles could potentially be used to differentiate IM-resistant from IM-responsive Ph+ patients (Figure 1A). Clustering analysis of expression of the selected oncogenic and tumor suppressing miRNAs differentially expressed between IM-resistant and IM-responsive $\mathrm{Ph}+$ patients clearly separates both groups suggesting that this miRNA profile could distinguish the different IM-response in CML patients (Figure 1B).

qRT-PCR validation. In order to validate the microarray data and to ensure that the variations observed were not due to a technical issue, we have carried out SYBR Green miRNA specific qRT-PCR analyses. We focused on a group of the selected oncogenic miRNAs with altered expression between IM-resistant and IM-responsive $\mathrm{Ph}+$ patients, $\mathrm{miR}-17$, miR18a, miR-20a, miR-21, miR-27a and miR-155, as well as on tumor suppressing miRNAs, let-7d, miR-205, miR-320, miR-451 and miR-574. Expression of each miRNA was determined in IM-resistant and IM-responsive $\mathrm{Ph}+$ patients and the data obtained was compared with that of healthy control individuals.

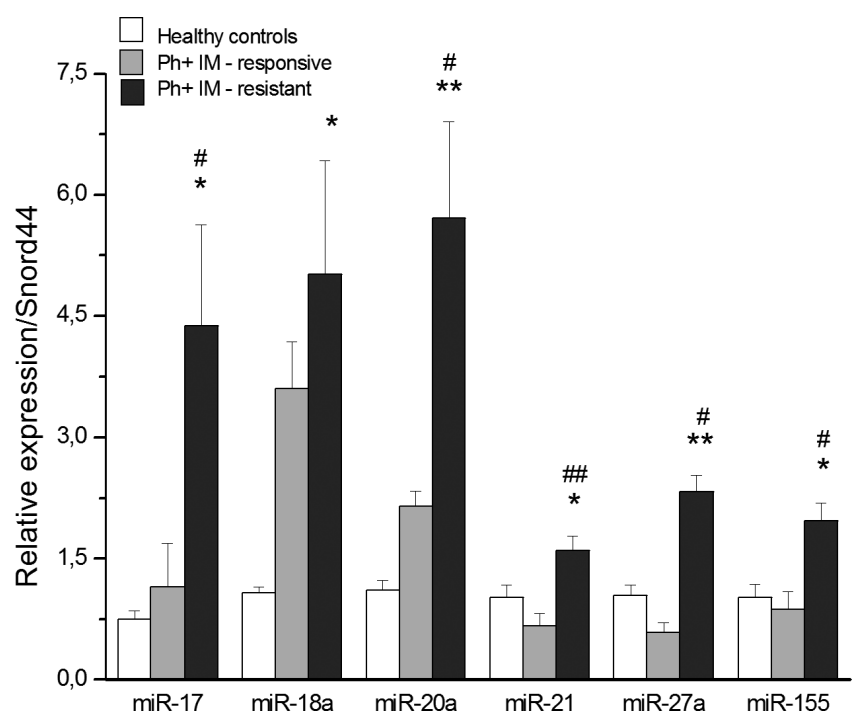

Figure 2. Real-time RT-PCR validation of the selected oncogenic miRNAs. The expression of miR-17, miR-18a, miR-20a, miR-21, miR-27a and miR155 was significantly up-regulated in IM-resistant $P h+$ patients $(n=4$, 3 replicates each, dark grey columns) compared to healthy individuals $(\mathrm{n}=14,3$ replicates each, white columns). Values represent mean \pm SEM; ${ }^{\star} p<0.05,{ }^{* *} p<0.01$. Significant increase of expression was observed in individual miRNAs between IM-resistant $(n=4,3$ replicates each, dark grey columns) and IM-responsive $P h+$ patients $(n=5,3$ replicates each, light grey columns). Values represent mean \pm SEM; \# $p<0.05$, \#\#p $<0.01$. All data were normalized against Snord 44 reference gene expression control. 


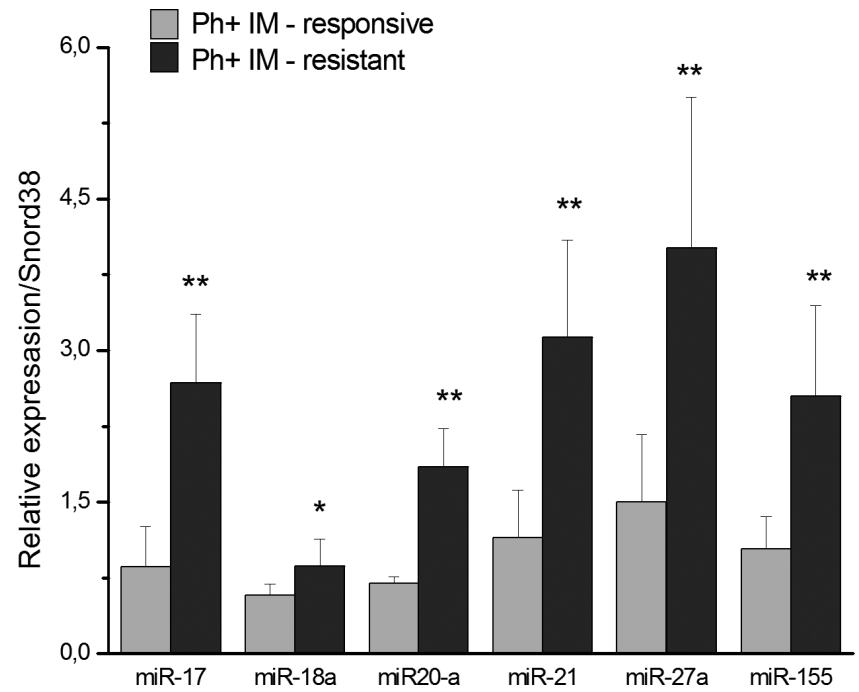

Figure 3. Real-time RT-PCR differential expression of oncogenic miRNAs in IM-resistant and IM-responsive patients. Expression of oncogenic miR-17, miR-18a, miR-20a, miR-21, miR-27a and miR-155 were significantly increased in IM-resistant ( $n=4,3$ replicates each, dark grey columns) compared to IM-responsive $\mathrm{Ph}+$ patients $(\mathrm{n}=5,3$ replicates each, light grey columns). IM treatment down-regulated oncogenic miRNAs in IM-responsive but not in IMresistant $\mathrm{Ph}+$ patients. Values represent mean $\pm \mathrm{SEM} ;{ }^{\star} \mathrm{p}<0.05,{ }^{* *} \mathrm{p}<0.01$. All data were normalized against Snord38B reference gene expression control.

qRT-PCR differential analysis of miRNA expression in IM-resistant $\mathrm{Ph}+$ patients compared to healthy individuals confirmed significantly increased expression levels of selected miRNAs, miR-17 (5.8-fold), miR-18a (4.6-fold), miR-20a (5.1-fold), miR-21 (1.7-fold), miR27a (2.3-fold) and miR-155 (2.0-fold), when normalized against endogenous reference gene expression control Snord44 (Figure 2). Significant increase of expression of miR-17 (3.8-fold), miR-18a (1.4-fold), miR-20a (2.7-fold), miR-21 (2.4-fold), miR-27a (4.0-fold) and miR-155 (2.3-fold) was also observed in IM-resistant $\mathrm{Ph}+$ patients when compared with $\mathrm{Ph}+\mathrm{IM}$-responsive ones (Figure 2). Similarly, significant up-regulation was confirmed when IM-resistant patients were compared to IM-responsive $\mathrm{Ph}+$ patients and data was normalized against different endogenous reference gene expression control Snord38. The observed increase of expression of individual miRNAs was following: miR-17 (3.1-fold), miR-18a (1.5-fold), miR-20a (2.6-fold), miR-21 (2.7-fold), miR-27a (2.7-fold) and miR-155 (2.5-fold) (Figure 3).

On the other hand, all selected tumor suppressing miRNAs except miR-451(showing only decreasing tendency) were significantly down-regulated after IM treatment in IM-resistant compared to IM-sensitive $\mathrm{Ph}+$ patients: let-7d (2.4-fold), miR-205 (1.9-fold), miR-320 (2.6-fold), miR-451 (1.1-fold) and miR-574 (2.4-fold). Obtained data were normalized against Snord 44 endogenous reference gene expression control (Figure 4).

Surprisingly, when these miRNAs were compared against healthy controls both IM- responsive and -resistant patients

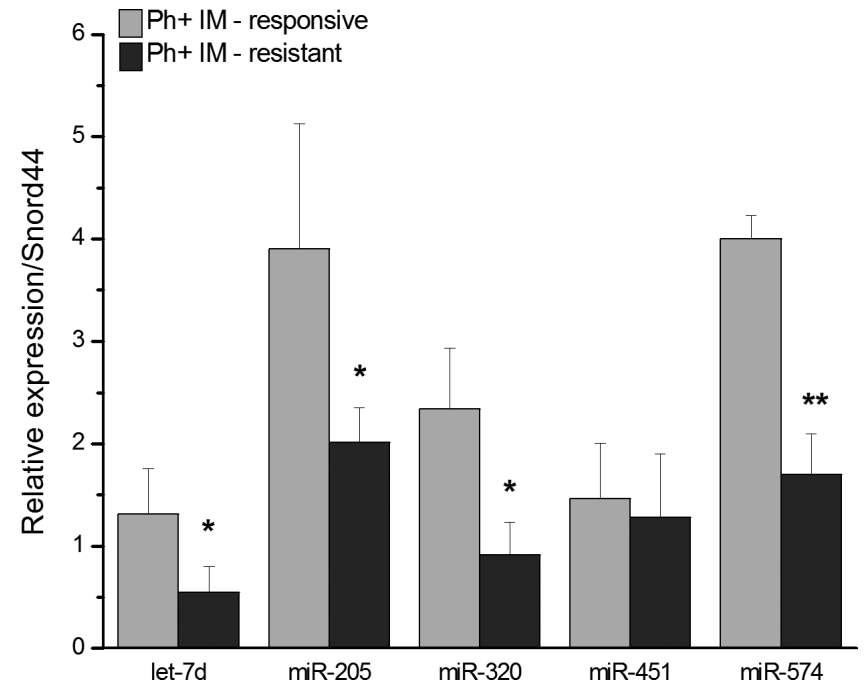

Figure 4. Real-time RT-PCR differential expression of selected tumor suppressor miRNAs in IM-resistant and IM-responsive patients. IM treatment increased the expression of let-7d, miR-205, miR-320, miR-451 and miR-574 in IM-responsive ( $n=5,3$ replicates each, dashed columns) but not in IM-resistant $\mathrm{Ph}+$ patients ( $\mathrm{n}=4,3$ replicates each, grey columns). Values represent mean $\pm \mathrm{SEM} ;{ }^{\star} \mathbf{p}<\mathbf{0 . 0 5},{ }^{\star *} \mathrm{p}<\mathbf{0 . 0 1}$. All data were normalized against Snord 44 reference gene expression control.

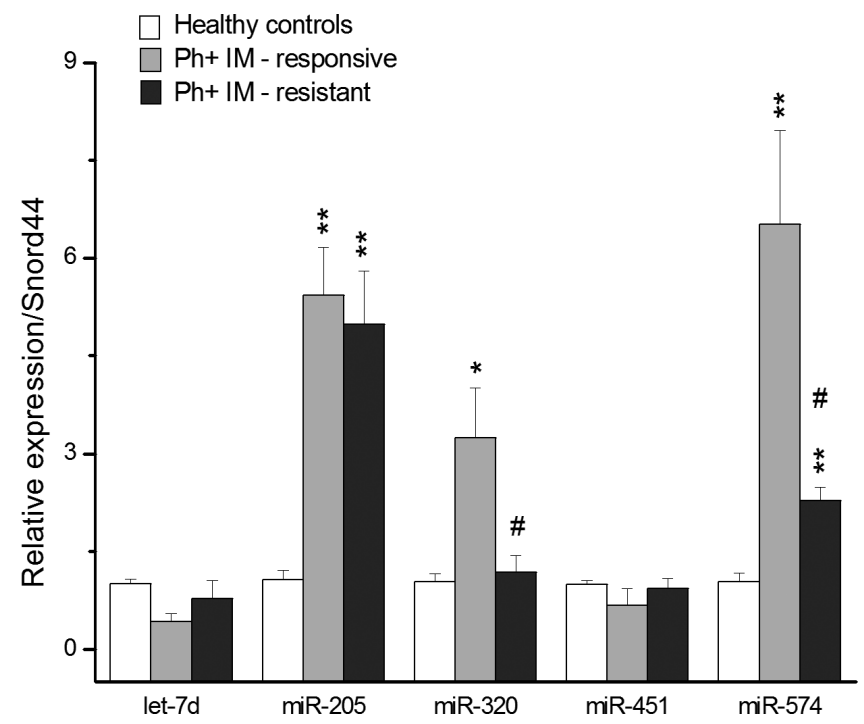

Figure 5. Real-time RT-PCR validation of the selected tumor suppressor miRNAs. In all patients, the expression of let-7d and miR-451 was similar to healthy controls and qRT-PCR validation showed no differences with respect to IM response. $\mathrm{miR}-205, \mathrm{miR}-320$ and miR-574 were significantly up-regulated in IM-responsive patients $(n=5,3$ replicates each, light grey columns). IM-resistant patients ( $n=4,3$ replicates each, dark grey columns) expressed higher levels of miR-205, miR-320 and miR-574 compared to healthy controls ( $n=14,3$ replicates each, white columns) but significantly lower compared to IM-responsive patients. Values represent mean $\pm \mathrm{SEM}$; ${ }^{*} \mathbf{p}<0.05,{ }^{* *} \mathrm{p}<0.01$ when compared against healthy individuals. Values represent mean $\pm S E M$; \# $<<0.05$, when responsive and resistant patients were compared. All data were normalized against Snord44 reference gene expression control. 
expressed up-regulated levels of miR-205 (5-fold in responsive, 4.7-fold in resistant), miR-320 (3.1-fold in responsive, 1.1 -fold in resistant) and miR-574 (6.2-fold in responsive, 2.2-fold in resistant), although the levels of these miRNAs were significantly higher in IM-responsive compared to -resistant patients. Expression levels of let-7d and miR-451 were unaffected (Figure 5).

In IM-responsive patients, IM treatment down-regulated expression levels of oncogenic miRNAs and up-regulated tumor suppressing miRNAs, while in IM-resistant patients the expression levels of oncogenic and tumor suppressing miRNAs stayed significantly increased and decreased respectively, even after 6 months of therapy.

\section{Discussion}

The current CML treatments include hydroxyurea, bone marrow transplantation or tyrosine kinase inhibitors (TKI). IM is commonly used highly effective TKI in CML treatment; however, IM resistance and intolerance remain a major clinical problem. 20-25\% of CML patients is resistant to IM or develops resistance during the treatment. The IM resistance may occur due to $B C R-A B L$ amplification, overexpression or spontaneous mutations in tyrosine kinase domain of $B C R-A B L$. Resistance to IM may also develop due to evolution of the disease with the occurrence of rare or novel cytogenetic aberrations leading to BCR-ABL independent proliferation of leukemic cells [26]. Regular monitoring of patients' resistance and practical monitoring of IM response can be used to guide treatment choice and provide patients with significantly better long-term outcomes. To predict and manage IM resistance, multiple potential biomarkers are intensively being searched. Recognition of IM intolerance and resistance, as well as monitoring of treatment outcomes, becomes even more difficult if patients carry rare variants of the $B C R-A B L$ fusion gene. In our study, we have compared miRNA expression profiles in small cohort of IM-resistant and IM-responsive CML patients carrying $\mathrm{Ph}$ chromosome with rare $B C R-A B L$ variants to identify additional molecular markers reflecting and predicting resistance to IM in these patients.

Microarray comparison of miRNA expression in peripheral blood of $\mathrm{Ph}+$ patients resistant to $\mathrm{IM}$ and $\mathrm{Ph}+$ patients responsive to this drug revealed 70 differentially expressed miRNAs, preferentially oncomiRs (miR-15a, miR-17, miR-18a, miR-20a, miR-21, miR-22, miR-26a, miR-27a, miR-29c, miR-93, miR145, miR-150, miR-155, miR-181a and miR-342) and several tumor suppressors (let-7, miR-34b, miR-205, miR-320, miR451 and miR-574). Of set of differentially expressed miRNAs, we further validated 6 up-regulated oncogenic miRNAs and 5 down-regulated suppressors that have been shown to be highly associated with pathogenesis of several hematological malignancies, including CML $[17,18]$.

Subsequently, significant up-regulation of these 6 oncomirs (miR-17, miR-18a, miR-20a, miR-21, miR-27a and miR-155) and down-regulation of 5 tumor suppressing miRNAs (let-7,
miR-205, miR-320, miR-451b and miR-574) have been validated by qRT-PCR. Both microarray and qRT-PCR validation confirmed that miR-17, miR-18a, miR-20a, miR-21, miR-27a and miR-155 are significantly up-regulated and let-7, miR-205, $\mathrm{miR}-320$, and $\mathrm{miR}-574$ are significantly down-regulated in IMresistant compared to IM-responsive $\mathrm{Ph}+$ patients. However, significant down-regulation of miR-451 was not confirmed by qRT-PCR, probably due to small cohort. Significantly different expression profiles of these miRNAs can distinguish IM-resistant form IM-responsive CML patients carrying rare $B C R-A B L$ transcripts.

miR-155 has been described to be overexpressed in many hematological malignancies $[27,28]$, being considered as a regulator of myelopoiesis [29]. However, its expression in CML remains rather controversial. Observed up-regulation of miR-155 in IM-resistant patients but not in IM-responsive patients seems to be in correlation with data of Rokah and co-authors [3] who detected down-regulation of miR-155 in chronic myeloid cells and CML patients. Overexpressed miR17 92 cluster plays an oncogenic role in CML, being regulated by c-MYC. $B C R-A B L$ fusion gene induces expression of cMYC and consequently up-regulates the cluster expression [30]. The miR-17 92 cluster contributes to proliferation and inhibition of apoptosis via E2F1 (E2F transcription factor 1), PTEN (phosphatase and tensin) and BIM (BCL2-interacting mediator of cell death) transcription factors; namely miR-17 and miR-20a has been described to inhibit pro-apoptotic BIM and subsequent mitochondria-dependent apoptosis [31]. Apoptotic and proliferative signals of c-MYC and E2F1 are tightly balanced by the expression of the miR-17 92 cluster [32-34]. In evolution, strongly conserved miR-21 has been found to be associated with a variety of neoplastic disorders targeting a number of tumor suppressor genes widely involved in growth, apoptosis, invasion and cell cycle (e.g. programmed cell death 4 [PDCD4], PTEN, tropomyosin [TPM1], etc.) [35, 36]. Knock-down of endogenous miR-21 expression contributed to sensitizing leukemic K562 cells to therapy mainly through increasing apoptosis [37]. Although overexpressed miR-21 is one of the most prominent miRNAs implicated in the tumor growth, proliferation, anti-apoptosis and response to chemotherapy, its role has rarely been characterized and studied with regard to CML [38]. miR-27a is an important regulator of hematopoietic development as it targets the 3' UTR of RUNX1 transcription factor, a master regulator in hematopoietic development [39]. Up-regulated miR-27a in cooperation with miR-17 92 cluster inhibits the RUNX1 and directly activates PU.1 and CEBPA (CCAAT/ enhancer-binding protein alpha), transcription factors critical for determination of the myeloid and lymphoid lineage from hematopoietic precursors [40-42]. Increased miR-27a also promotes angiogenesis [40] and can alter the activity of NF- $\kappa B, M A P K s / p 38$, c-Jun and ERK.

In correlation with published data, we have also observed down-regulation of typical tumor suppressing miRNAs: let-7, miR-34 and miR-200 families [43, 44]. Several studies have re- 
ported that down-regulation of tumor suppressing miRNAs is involved in regulation of apoptosis, epithelial to mesenchymal transition (EMT) and tumor invasion in association with CML and other hematological malignancies [23, 45-47]. Observed down-regulated miR-320 via posttranscriptional repression of TfR-1 (transferrin receptor-1) effects cell growth modulating iron availability, and targets other proteins necessary for cell growth and proliferation [48]. Down-regulated miR-451 and miR-547 are considered potent suppressors of oncogenesis that have been reported to target several signaling pathways $[37,49]$. miR-451 together with miR-27a has been described to modulate multidrug resistance by targeting BCL2 and MDR1, respectively [50].

According to our results that are in line with observations of other authors, we suggest that oncogenic miRNAs, miR155, miR17 92 cluster together with miR-21 and miR-27a, and tumor suppressing miRNAs, let-7, miR-205, miR-320, miR-451 and miR-574, could represent a suitable group of biomarkers relevant for early detection of IM-resistance and CML pathophysiology. Thus, these selected miRNAs represent novel molecular biomarkers offering additional molecular diagnostic and predictive tool enabling efficient monitoring of treatment response and therapy outcomes in a group of CML patients with rare $B C R-A B L$ variants. Undoubtedly, to confirm predictive potential of identified miRNA biomarkers, these should thereto be examined in samples from patients with rare $B C R-A B L$ variants before receiving a therapy. Such experiments are to be carried out in the laboratory in near future.

Acknowledgements: This work was supported by grant co-sponsored by sources of EU - European fund for regional development through operation program of Research and Development (project code ITMS 26240220074/call code OPVaV-2011/4.2/07-SORO). We would like to greatly acknowledge personnel of University Hospital of Medical Faculty, Comenius University and National Institute for Oncology, in Bratislava, Slovakia for their contribution and help with patients`samples collection and management.

\section{References}

[1] DRUKER BJ, GUILHOT F, O'BRIEN SG, GATHMANN I, KANTARJIAN $\mathrm{H}$ et al. Five-year follow-up of patients receiving imatinib for chronic myeloid leukemia. N Engl J Med 2006; 355: 2408-2417. http://dx.doi.org/10.1056/NEJMoa062867

[2] MINEO M, GARFIELD SH, TAVERNA S, FLUGY A, DE LEO $\mathrm{G}$ et al. Exosomes released by K562 chronic myeloid leukemia cells promote angiogenesis in a Src-dependent fashion. Angiogenesis 2012; 15: 33-45. http://dx.doi.org/10.1007/ $\underline{\text { s10456-011-9241-1 }}$

[3] ROKAH OH, GRANOT G, OVCHARENKO A, MODAI S, PASMANIK-CHOR M et al. Downregulation of miR-31, miR-155, and miR-564 in chronic myeloid leukemia cells. PLoS One 2012; 7: e35501. http://dx.doi.org/10.1371/journal. pone. 0035501

[4] BHUTRA S, LENKALA D LACROIX B, YE M, HUANG RS. Identifying and validating a combined mRNA and microRNA signature in response to imatinib treatment in a chronic myeloid leukemia cell line. PLoS One 2014; 9: e115003. http:// dx.doi.org/10.1371/journal.pone.0115003

[5] MELO JV. BCR-ABL gene variants. Baillieres Clin Haematol 1997; 10: 203-222. http://dx.doi.org/10.1016/S0950-3536(97) $80003-0$

[6] TABASSUM N, SABOOR M, GHANI R AND MOINUDDIN $M$. Heterogeneity of BCR-ABL rearrangement in patients with chronic myeloid leukemia in Pakistan Pak J Med Sci 2014; 30 : 850-853.

[7] COSTINEAN S, ZANESI N, PEKARSKY Y, TILI E, VOLINIA $S$ et al. Pre-B cell proliferation and lymphoblastic leukemia/ high-grade lymphoma in $\mathrm{E}(\mathrm{mu})$-miR155 transgenic mice. Proc Natl Acad Sci U S A 2006; 103: 7024-7029. http://dx.doi. org/10.1073/pnas.0602266103

[8] HATFIELD S, RUOHOLA-BAKER H. microRNA and stem cell function. Cell and tissue research 2008; 331: 57-66. http:// dx.doi.org/10.1007/s00441-007-0530-3

[9] CHEN Y, STALLINGS RL. Differential patterns of microRNA expression in neuroblastoma are correlated with prognosis, differentiation, and apoptosis. Cancer Res 2007; 67: 976-983. http://dx.doi.org/10.1158/0008-5472.CAN-06-3667

[10] BALTIMORE D, BOLDIN MP, O'CONNELL RM, RAO DS, TAGANOV KD. MicroRNAs: new regulators of immune cell development and function. Nat Immunol 2008; 9: 839-845. http://dx.doi.org/10.1038/ni.f.209

[11] TRIBOULET R, MARI B, LIN YL, CHABLE-BESSIA C, BENNASSER Y et al. Suppression of microRNA-silencing pathway by HIV-1 during virus replication. Science 2007; 315: 1579-1582. http://dx.doi.org/10.1126/science.1136319

[12] KASINSKI AL, SLACK FJ. Epigenetics and genetics. MicroRNAs en route to the clinic: progress in validating and targeting microRNAs for cancer therapy. Nature reviews Cancer 2011; 11: 849-864. http://dx.doi.org/10.1038/nrc3166

[13] RAMKISSOON SH, MAINWARING LA, OGASAWARA Y, KEYVANFAR K, MCCOY JP et al. Hematopoietic-specific microRNA expression in human cells. Leuk Res 2006; 30: 643-647. http://dx.doi.org/10.1016/j.leukres.2005.09.001

[14] CALIN GA, DUMITRU CD, SHIMIZU M, BICHI R, ZUPO $\mathrm{S}$ et al. Frequent deletions and down-regulation of micro- RNA genes miR15 and miR16 at 13q14 in chronic lymphocytic leukemia. Proc Natl Acad Sci U S A 2002; 99: 15524-15529. http://dx.doi.org/10.1073/pnas.242606799

[15] FULCI V, CHIARETTI S, GOLDONI M, AZZALIN G, CARUCCI $N$ et al. Quantitative technologies establish a novel microRNA profile of chronic lymphocytic leukemia. Blood 2007; 109: 4944-4951. http://dx.doi.org/10.1182/blood-2006 $\underline{-12-062398}$

[16] HE L, THOMSON JM, HEMANN MT, HERNANDOMONGE E, MU D et al. A microRNA polycistron as a potential human oncogene. Nature 2005; 435: 828-833. http://dx.doi.org/10.1038/nature03552

[17] HAYASHITA Y, OSADA H, TATEMATSU Y, YAMADA H, YANAGISAWA $\mathrm{K}$ et al. A polycistronic microRNA cluster, miR-17-92, is overexpressed in human lung cancers and enhances cell proliferation. Cancer Res 2005; 65: 9628-9632. http://dx.doi.org/10.1158/0008-5472.CAN-05-2352 
[18] RINALDI A, PORETTI G, KWEE I, ZUCCA E, CATAPANO CV et al. Concomitant MYC and microRNA cluster miR-17-92 (C13orf25) amplification in human mantle cell lymphoma. Leuk Lymphoma 2007; 48: 410-412. http://dx.doi. org/10.1080/10428190601059738

[19] KLUIVER J, POPPEMA S, DE JONG D, BLOKZIJL T, HARMS G et al. BIC and miR-155 are highly expressed in Hodgkin, primary mediastinal and diffuse large B cell lymphomas. J Pathol 2005; 207: 243-249. http://dx.doi. org/10.1002/path. 1825

[20] EIS PS, TAM W, SUN L, CHADBURN A, LI Z et al. Accumulation of miR-155 and BIC RNA in human B cell lymphomas. Proc Natl Acad Sci U S A 2005; 102: 3627-3632. http://dx.doi. org/10.1073/pnas.0500613102

[21] LANDGRAF P, RUSU M, SHERIDAN R, SEWER A, IOVINO $\mathrm{N}$ et al. A mammalian microRNA expression atlas based on small RNA library sequencing. Cell 2007; 129: 1401-1414. http://dx.doi.org/10.1016/j.cell.2007.04.040

[22] O'CONNELL RM, RAO DS, CHAUDHURI AA, BOLDIN MP, TAGANOV KD et al. Sustained expression of microRNA155 in hematopoietic stem cells causes a myeloproliferative disorder. The Journal of experimental medicine 2008; 205: 585-594. http://dx.doi.org/10.1084/jem.20072108

[23] THOMPSON RC, HERSCOVITCH M, ZHAO I, FORD TJ, GILMORE TD. NF-kappaB down-regulates expression of the B-lymphoma marker CD10 through a miR-155/PU.1 pathway. J Biol Chem 2011; 286: 1675-212682. http://dx.doi. org/10.1074/jbc.M110.177063

[24] VAN DEN BERG A, KROESEN BJ, KOOISTRA K, DE JONG D, BRIGGS J et al. High expression of B-cell receptor inducible gene BIC in all subtypes of Hodgkin lymphoma. Genes Chromosomes Cancer 2003; 37: 20-28. http://dx.doi.org/10.1002/ gcc. 10186

[25] POSPISIL V, VARGOVA K, KOKAVEC J, RYBAROVA J, SAVVULIDI $F$ et al. Epigenetic silencing of the oncogenic miR-17-92 cluster during PU.1-directed macrophage differentiation. The EMBO journal 2011; 30: 4450-4464. http:// dx.doi.org/10.1038/emboj.2011.317

[26] HOCHHAUS A, KREIL S, CORBIN AS, LA ROSEE P, MULLER MC et al. Molecular and chromosomal mechanisms of resistance to imatinib (STI571) therapy. Leukemia 2002; 16: 2190-2196. http://dx.doi.org/10.1038/sj.leu.2402741

[27] ELTON TS, SELEMON H, ELTON SM, PARINANDI NL. Regulation of the MIR155 host gene in physiological and pathological processes. Gene 2013; 532: 1-12. http://dx.doi. org/10.1016/j.gene.2012.12.009

[28] JURKOVICOVA D, MAGYERKOVA M, KULCSAR L, KRIVJANSKA M, KRIVJANSKY V et al. miR-155 as a diagnostic and prognostic marker in hematological and solid malignancies. Neoplasma 2014; 61:241-251. http://dx.doi.org/10.4149/ neo $2014 \quad 032$

[29] FARAONIA I, ANTONETTI FR, CARDONE J, BONMASSAR E. miR-155 gene: a typical multifunctional microRNA. Biochim Biophys Acta 2009; 1792: 497-505. http://dx.doi. org/10.1016/j.bbadis.2009.02.013

[30] VENTURINI L, BATTMER K, CASTOLDI M, SCHULTHEIS B, HOCHHAUS A et al. Expression of the miR-17-92 polycis- tron in chronic myeloid leukemia (CML) CD34+ cells. Blood 2007; 109: 4399-4405. http://dx.doi.org/10.1182/blood-2006 $\underline{-09-045104}$

[31] WENG H, HUANG H, DONG B, ZHAO P, ZHOU H et al. Inhibition of miR-17 and miR-20a by oridonin triggers apoptosis and reverses chemoresistance by derepressing BIM-S. Cancer Res 2014; 74: 4409-4419. http://dx.doi.org/10.1158/00085472.CAN-13-1748

[32] O`DONNELL KA, WENTZEL EA, ZELLER KI, DANG CV, MENDELL JT. c-Myc-regulated microRNAs modulate E2F1 expression. Nature 2005; 435: 839-843. http://dx.doi. org/10.1038/nature03677

[33] SYLVESTRE Y, DE GUIRE V, QUERIDO E, MUKHOPADHYAY UK, BOURDEAU V et al. An E2F/miR-20a autoregulatory feedback loop. J Biol Chem 2007; 282: 2135 2143. http://dx.doi.org/10.1074/jbc.M608939200

[34] WOODS K, THOMSON JM, HAMMOND SM. Direct regulation of an oncogenic micro-RNA cluster by E2F transcription factors. J Biol Chem 2007; 282: 2130-2134. http://dx.doi. org/10.1074/jbc.C600252200

[35] SELCUKLU SD, DONOGHUE MT, SPILLANE C. miR-21 as a key regulator of oncogenic processes. Biochem Soc Trans 2009; 37: 918-925. http://dx.doi.org/10.1042/BST0370918

[36] LANKAT-BUTTGEREIT B, GOKE R. The tumour suppressor Pdcd4: recent advances in the elucidation of function and regulation. Biology of the cell / under the auspices of the European Cell Biology Organization 2009; 101: 309-317. http://dx.doi.org/10.1042/BC20080191

[37] LI X, SANDA T, LOOK AT, NOVINA CD, VON BOEHMER H. Repression of tumor suppressor miR-451 is essential for NOTCH1-induced oncogenesis in T-ALL. The Journal of experimental medicine 2011; 208: 663-675. http://dx.doi. org/10.1084/jem.20102384

[38] LAWRIE CH. MicroRNAs and lymphomagenesis: a functional review. Br J Haematol 2013; 160: 571-581. http://dx.doi. org/10.1111/bjh.12157

[39] FRIEDMAN AD. Cell cycle and developmental control of hematopoiesis by Runx1. Journal of cellular physiology 2009; 219: 520-524. http://dx.doi.org/10.1002/jcp. 21738

[40] HUANG G, ZHANG P, HIRAI H, ELF S, YAN X et al. PU.1 is a major downstream target of AML1 (RUNX1) in adult mouse hematopoiesis. Nat Genet 2008; 40: 51-60. http:// dx.doi.org/10.1038/ng.2007.7

[41] GUO H, MA O, SPECK NA, FRIEDMAN AD. Runx1 deletion or dominant inhibition reduces Cebpa transcription via conserved promoter and distal enhancer sites to favor monopoiesis over granulopoiesis. Blood 2012; 119: 4408-4418. http://dx.doi.org/10.1182/blood-2011-12-397091

[42] ROSSETTI S, SACCHI N. RUNX1: A MicroRNA Hub in Normal and Malignant Hematopoiesis. International journal of molecular sciences 2013; 14: 1566-1588. http://dx.doi. org/10.3390/ijms14011566

[43] ZHANG W, DAHLBERG JE, TAM W. MicroRNAs in tumorigenesis: a primer. Am J Pathol 2007; 171: 728-738. http:// dx.doi.org/10.2353/ajpath.2007.070070

[44] JONGEN-LAVRENCIC M, SUN SM, DIJKSTRA MK, VALK PJ, LOWENBERG B. MicroRNA expression profiling in rela- 
tion to the genetic heterogeneity of acute myeloid leukemia. Blood 2008; 111: 5078-5085. http://dx.doi.org/10.1182/blood$\underline{\text { 2008-01-133355 }}$

[45] LODYGIN D, TARASOV V, EPANCHINTSEV A, BERKING C, KNYAZEVA $\mathrm{T}$ et al. Inactivation of miR-34a by aberrant $\mathrm{CpG}$ methylation in multiple types of cancer. Cell Cycle 2008; 7: 2591-2600. http://dx.doi.org/10.4161/ cc.7.16.6533

[46] WIKLUND ED, BRAMSEN JB, HULF T, DYRSKJOT L, RAMANATHAN R et al. Coordinated epigenetic repression of the miR-200 family and miR-205 in invasive bladder cancer. Int J Cancer 2011; 128: 1327-1334. http://dx.doi.org/10.1002/ ijc. 25461

[47] DING XM. MicroRNAs: regulators of cancer metastasis and epithelial-mesenchymal transition (EMT). Chinese journal of cancer 2014; 33: 140-147. http://dx.doi.org/10.5732/ cjc.013.10094

[48] SCHAAR DG, MEDINA DJ, MOORE DF, STRAIR RK, TING Y. miR-320 targets transferrin receptor 1 (CD71) and inhibits cell proliferation. Experimental hematology 2009; 37: 245-255. http://dx.doi.org/10.1016/j.exphem.2008.10.002

[49] CHIYOMARU T, YAMAMURA S, FUKUHARA S, HIDAKA $\mathrm{H}, \mathrm{MAJID} S$ et al. Genistein up-regulates tumor suppressor microRNA-574-3p in prostate cancer. PLoS One 2013; 8: e58929. http://dx.doi.org/10.1371/journal.pone.0058929

[50] ZHU H, WU H, LIU X, EVANS BR, MEDINA DJ et al. Role of MicroRNA miR-27a and miR-451 in the regulation of MDR1/P-glycoprotein expression in human cancer cells. Biochem Pharmacol 2008; 76: 582-588. http://dx.doi. org/10.1016/j.bcp.2008.06.007 